watering of roadways during the mining operation. Employee education on health effects of dust exposure, work practices, maintenance of equipment and PPE, medical surveillance, chest $\mathrm{X}$-rays and $\mathrm{TB}$ awareness and monitoring are essential in the elimination of silicosis.

\section{PASSIVE MONITORING OF CHEMICAL EXPOSURES IN SOUTH FLORIDA FIREFIGHTERS USING SILICONE WRISTBANDS}

${ }^{1}$ Katerina M Santiago*, 'Alberto J Caban-Martinez, '1Jeramy Baum, 'Johnathan Pangborn, ${ }^{2}$ Emre Dikici, ${ }^{1}$ Natasha Shaefer Solle, ${ }^{3}$ David A Sterling, ${ }^{1}$ Kevin Moore, ${ }^{1}$ Kathy Grace, ${ }^{2}$ Sylvia Daunert, ${ }^{2}$ Sapna Deo, ${ }^{1}$ Erin N Kobetz. 'Department of Public Health Sciences, University of Miami, Miller School of Medicine, Miami, FL, USA; ${ }^{2}$ Department of Biochemistry and Molecular Biology, University of Miami, Miller School of Medicine, Miami, FL, USA; ${ }^{3}$ School of Public Health, University of North Texas Health Science Centre, Fort Worth, TX

\subsection{6/oemed-2018-ICOHabstracts.574}

Introduction Firefighters are likely to be exposed to many toxic chemicals in the performance of their work duties such as polycyclic aromatic hydrocarbons (PAHs). Chemical exposures may occur through dermal, oral, or inhalation pathways. Passive sampling devices are used to sequester organic molecules through passive diffusion and provide time-weighted averages of chemical concentrations. This pilot study uses silicone-based wristbands as a personal passive sampler to detect known carcinogens during a 24 hour work shift.

Methods Twenty-four wristbands were deployed across various fire services throughout South Florida. Prior to deployment, bands were cleaned using a standardised cleaning protocol to remove contamination and optimise the surface for absorption. Wristbands were then packaged in air-tight bags to prevent contamination. Wristbands were worn on fire service personnel and collected at the end of a 24 hour work shift. Chemical contaminants were then extracted from the wristband and analysed for PAHs-identified using the EPA IRIS, California Proposition 65, and IRAC datasets-using gas chromatography-mass spectrometry.

Results The average number of chemicals found across all wristbands $(n=24)$ was 23 with 4 categorised as carcinogenic to humans (i.e., Benzo[b]fluoranthene, Benzo[j]fluoranthene, Chrysene, and Naphthalene). All bands had at least one PAH present, specifically, $87.5 \%$ contained Benzo[b]fluoranthene (mean $=5.23 \mathrm{ng} / \mathrm{band}$ ), $50 \%$ contained Benzo[j]fluoranthene (mean=2.05 ng/band), 79.2\% contained Chrysene (mean=9.55 ng/band), and 100\% contained Napthalene (mean=176.53 ng/ band). Actual types of exposure compounds is likely to be larger than the observed data as the group of PAHs detected was limited to three existing datasets.

Discussion Silicone-based wristbands are feasible to use within the fire service to detect and characterise ambient hazardous chemical compounds. These personal self-samplers used during a 24 hour collection period identified various PAHs in the firefighter work environment. Objective measures of harmful chemical exposures in the fire service should be monitored with a comprehensive surveillance system that includes personal sampler devices.
967 EVALUATING TEMPERATURE CHANGES AND VOLATILE
ORGANIC COMPOUND OFF-GASSING IN TURNOUT
PROTECTIVE GEAR ENSEMBLES AMONG FLORIDA
FIREFIGHTERS

${ }^{1}$ Katerina M Santiago*, 'Alberto J Caban-Martinez, ${ }^{1}$ Jeramy Baum, ${ }^{1} J o h n a t h a n$ Pangborn, ${ }^{2}$ Emre Dikici, ${ }^{1}$ Natasha Shaefer Solle, 'David Sterling, 'Tualy Koru-Sengul, 'Kevin Moore, ${ }^{1}$ Isabelle Salvaterra, ${ }^{2}$ Sylvia Daunert, ${ }^{2}$ Sapna Deo, ${ }^{1}$ Erin N Kobetz. ${ }^{1}$ Department of Public Health Sciences, University of Miami, Miller School of Medicine, Miami, FL, USA; ${ }^{2}$ Department of Biochemistry and Molecular Biology, University of Miami, Miller School of Medicine, Miami, FL, USA

\subsection{6/oemed-2018-ICOHabstracts.575}

Introduction Firefighter protective gear ensembles have been shown in controlled laboratory and staged live fire training experiments, to collect and harbour carcinogens such as polycyclic aromatic hydrocarbons (PAHs) and volatile organic compounds (VOCs). Protective gear is often time transported in the personal vehicle of firefighters, resulting in cross-contamination between the vehicle and the fire incident environment. In the Southern United States particularly, ambient warmer temperatures may influence the rate of VOC gear off-gassing. This pilot study characterises temperature and particle off-gassing of firefighter turnout gear immediately following a 24 hour work shift.

Methods Twelve sets of gear were obtained from South Florida career firefighters. Their protective gear, including helmet, gloves, hood, pants, boots and turnout coat, were placed in a large vacuum sealed Pelican case immediately after a 24 hour work shift. Turn-out gear was randomly selected at each fire station regardless of fire exposure. A photoionization gas detector (0.2 to $200 \mathrm{ppm})$, MetOne particle counter, Chromosorb diffusion patch, and a temperature logger were placed in each case with the ensemble for a 24 hour collection period.

Results In two extreme observation points, VOC off-gassing was moderately, but significantly, correlated with temperature changes within the exposed gear (case\#1: $\mathrm{r}=0.50 ; \mathrm{p}<0.001$ ) while a low correlation was observed in case\#2 $(\mathrm{r}=0.06$; $\mathrm{p}=0.01)$. Fine particulate matter $(1-10 \mu \mathrm{m})$ was documented at least up to 1 hour after the gear was placed in most cases. Smaller size $(0.3-0.5 \mu \mathrm{m})$ particulate matter was present up to 5 hours after placement across several cases.

Discussion Firefighter turnout gear used during real-life fire incident response events was documented to release VOCs and particles immediately after a 24 hour work shift. These results suggest the importance of the development of robust decontamination procedures immediately following a fire incident response is needed to reduce exposure to potential carcinogens from firefighter protective gear.

\section{MODIFICATION AND IMPROVEMENT OF FIT TEST METHOD USING AMBIENT AEROSOLS}

${ }^{1} \mathrm{SH}$ Yang, ${ }^{1} \mathrm{SH}$ Huang, ${ }^{2} \mathrm{YM}$ Kuo, ${ }^{1} \mathrm{KJ}$ Yang, ${ }^{3} \mathrm{CY}$ Lai, ${ }^{1} \mathrm{CC}$ Chen*. ${ }^{1}$ National Taiwan University, Taipei, Taiwan; ${ }^{2}$ Chung Hwa University of Medical Technology, Tainan, Taiwan; ${ }^{3}$ Chung Shan Medical University, Taichung, Taiwan

\subsection{6/oemed-2018-ICOHabstracts.576}

Introduction Fit testing should be performed before the use of tight-fitting respirators. However, it may not always be 
conducted for various reasons, mostly time consuming and costly. This study aimed to shorten the fit testing procedures by improving the instrumental settings, sampling system design, and data analysis protocols.

Methods Experiments of fit factor measurements were divided into two parts: constant flow and cyclic flow using a breathing simulator. To simulate leakage, capillaries $(10 \mathrm{~mm}$ in length, diameter $1.0-1.5 \mathrm{~mm}$ ) were used to insert on N95 and N100 filtering facepieces. The ratio of total to leak flow was considered the 'true fit factor, FFt'. Flow rates ranging from $5-50 \mathrm{~L} / \mathrm{min}$ were employed to study the flow dependency. The measured fit factors were determined by concurrent particle concentration measured by a Portacount and a OPS 3330. The default $1.7 \mathrm{~m}$ sampling tube was used to connect filtering facepiece to the aerosol instruments. In addition, the effects of breathing pattern (tidal volume: $0.5-1 \mathrm{~L}$, frequency: 5-20 times/min) and lung deposition (with/without HEPA filter behind the respirator) on in-mask particle concentration during fit testing were analysed, to explore the minimal sampling time that approximated the FFt.

Results The particle measurement response times for Portacount and OPS were approximately 5 and $2 \mathrm{~s}$, respectively. For P100 respirators, most measured fit factors were close to the FFt. Whereas, there was an underestimation while using N95 respirator due to filter penetration. Therefore, N95-companion was necessary while testing N95 respirator. For the cyclic flow tests, the fit factor was overestimated because the sampling tube was connected onto the facepiece where filtered air was partly sampled. The higher the breathing flow rate, the more the fit factor was overestimated. On the other hand, the measured fit factor would be close to the FFt when using the highest concentration during a breathing cycle (FFmin). In theory, it could be decided in only one breathing cycle.

Conclusion With improved design in instrumental setting and operating procedures, a fit test for an individual exercise would take approximately only $12 \mathrm{~s}$. Therefore, the whole fit testing process could be shortened from 7.5 to about $3 \mathrm{~min}$.

\section{DEVELOPMENT OF A COOL AND CLEAN AIR MOTORCYCLE HELMETS}

${ }^{1} \mathrm{AL}$ Jian*, ${ }^{1} \mathrm{SH}$ Huang, ${ }^{2} \mathrm{YM}$ Kuo, ${ }^{1} \mathrm{CW}$ Lin, ${ }^{1} \mathrm{WC}$ Lee, ${ }^{1} \mathrm{CC}$ Chen. ${ }^{1}$ National Taiwan University, Taipei, Taiwan; ${ }^{2}$ Chung Hwa University of Medical Technology, Tainan, Taiwan

\subsection{6/oemed-2018-ICOHabstracts.577}

Introduction Motorcyclists could be exposed to high $\mathrm{PM}_{2.5}$ up to $460 \mu \mathrm{g} / \mathrm{m}^{3}$. The aim of this work was to develop a full faced helmet $(\mathrm{FFH})$ that provides clean air and cool temperature inside the helmet to reduce particulate exposure and increase comfort for motorcyclists.

Methods A commercial FFH was modified to generate cool and clean air in a way similar to the powered-air-purified-respirator, commonly used in industrial settings. Three different clean air supply locations (A: upper rear of the head, B: zygomatic side, and C: lower chin) were applied to investigate the location effect. A small wind tunnel was used to simulate the turbulence that motorcyclists might encounter while riding on the road. The operating parameters included: the supply air flow rate to the helmet $\left(\mathrm{Q}_{\mathrm{s}}\right)$, the velocity in the wind tunnel $\left(\mathrm{U}_{0}\right)$ and breathing flow rate which is a combination of tidal volume and breathing frequency. To minimise infiltration of aerosol outside the helmet into the breathing zone, the FFH was tightly surrounded with a neckerchief made of different materials. The stiffness of the neckerchief was measured by using a $45^{\circ}$ angled Cantilever. A condensation particle counter was used to measure particle number concentrations both inside and outside the FFH, to calculate the protection factor, PF.

Results The PF of the FFH increased with increasing $Q_{s}$, but decreased with increasing wind speed and breathing flow rate. At breathing flow rate of $7.5 \mathrm{~L} / \mathrm{min}$ with the $\mathrm{FFH}$ sealed using neckerchief, PF increased from 1 to 900 as Qs increased from 0 to $50 \mathrm{~L} / \mathrm{min}$ under calm air condition, with air supply through location A. Meanwhile, the PF decreased from 900 to 3 when wind velocity increased from calm air to $10 \mathrm{~m} / \mathrm{s}$. The PF increased significantly when the FFH was sealed with a neckerchief made of soft shell (stiffness $19.2 \mathrm{~mm}, 28 \mathrm{~cm}$ long). In addition, the temperature on the top of head decreased with increasing supply air flow.

Discussion Applying a higher $\mathrm{Q}_{\mathrm{s}}$ up to $100 \mathrm{~L} / \mathrm{min}$ is necessary, not only to maintain a positive pressure inside the helmet but also to decrease the concentration of carbon dioxide exhaled by the wearer. The use of the soft shell neckerchief is also critical to reduce the infiltration due to external turbulence.

\section{SHIFT OF AEROSOL PENETRATION IN SIZE-SELECTIVE CYCLONE SAMPLERS}

${ }^{1} \mathrm{TJ}$ Chen, ${ }^{1} \mathrm{SH}$ Huang, ${ }^{1} \mathrm{CW}$ Lin, ${ }^{2} \mathrm{YM}$ Kuo, ${ }^{1} \mathrm{CC}$ Chen. ${ }^{1}$ National Taiwan University, Taipei, Taiwan; ${ }^{2}$ Chung Hwa University of Medical Technology, Tainan, Taiwan

\subsection{6/oemed-2018-ICOHabstracts.578}

Introduction Size-selective aerosol samplers are used to assess the health effect, because the retention of deposited particles in the respiratory tract is strongly size dependent. The main objective of this work is to examine the particle loading effects on PM2.5 cyclone samplers.

Methods In this work, five PM2.5 cyclones of different body diameters $(9.3-35.6 \mathrm{~mm})$, derived from the BGI VSCC, were designed and fabricated to investigate the effects of particle loading. An ultrasonic atomizing nozzle was used to generate micro-meter-sized potassium sodium tartrate (PST) particles and Sodium chloride $(\mathrm{NaCl})$ particles as solid challenge aerosols, and di-ethyl-hexyl-sebacate (DEHS) particles as liquid challenge particles. Aerosol number size distributions and concentrations, both upstream and downstream of the cyclones, were measured using an aerodynamic particle sizer. In addition to the cyclone body diameter, other parameters investigated in this work included: challenge aerosol size distribution, chamber humidity, and the material of the cyclone.

Results The PM2.5 cyclones could be used to sample liquid particles without any bias, because the deposited liquid aerosols dripped down and did not accumulate on the inner wall of the cyclone. However, when challenged with solid particles, the deposited and accumulated aerosols on the wall reduced the aerosol penetration, and changed the curve to be less sharp. The extent of underestimation was affected by many parameters, such as challenge aerosol size distribution, humidity, test agent, and the elastic properties of the cyclone and the test agent. On average, there was an underestimation of $20 \%$ of $2.5 \mu \mathrm{m}$ aerosol penetration when challenged with PST particles, regardless of cyclone body size. This suggested that cyclones might not be ideal for sampling solid particles.

Discussion Cyclone samplers currently used for size-selective sampling are likely subject to aerosol loading effect, and resulted in underestimation of the PM2.5 measurements. The use of virtual cyclone or wet cyclone might solve parts of the problem. 